\title{
Aplicação da teoria de preços hedônicos para avaliação da influência da caminhabilidade no preço de venda de imóveis residenciais
}

\author{
Shanna Trichês Lucchesi ${ }^{1}$, Ana Margarita Larranaga Uriarte ${ }^{2}$, Helena Beatriz Bettella Cybis ${ }^{3}$ \\ ${ }_{1}^{1}$ Departamento de Engenharia de Produção e Transportes, UFRGS, Brasil, slucchesi@gmail.com \\ 2Departamento de Engenharia de Produção e Transportes, UFRGS, Brasil, analarra@producao.ufrgs.br \\ ${ }^{3}$ Departamento de Engenharia de Produção e Transportes, UFRGS, Brasil, helenabc@producao.ufrgs.br
}

\section{Recebido:}

15 de março de 2018

Aceito para publicação:

13 de maio de 2018

Publicado:

4 de novembro de 2018

Editor de área:

Cira Pitombo, EESC-USP

\section{Palavras-chaves:}

Caminhabilidade;

Preços hedônicos;

Equações estruturais.

\section{Keywords:}

Walkability;

Hedonic price theory;

Structural equation model.

DOI:10.14295/transportes.v26i3.1628

OPEN 0 Access

\begin{abstract}
RESUMO
O objetivo deste trabalho é avaliar a influência da caminhabilidade no preço de venda de imóveis residenciais, através de um estudo de caso na cidade do Rio de Janeiro. As análises foram conduzidas através de uma abordagem hedônica, utilizando modelos de equações estruturais para construções das variáveis latentes de características não observáveis. Nesse caso enquadra-se a caminhabilidade e a segurança pública, inclusas no modelo testado. Essa metodologia possibilitou a análise da estrutura das variáveis latentes, suas relações mútuas e com o preço dos imóveis. Os resultados obtidos mostram que o preço do metro quadrado de imóveis residenciais à venda na área de estudo cresce conforme aumenta a caminhabilidade. A segurança pública, a declividade viária e a presença de construções aprazíveis foram identificadas como os fatores mais importantes na explicação da caminhabilidade e, consequentemente, na valorização do imóvel.
\end{abstract}

\begin{abstract}
The aim of this study is to evaluate the influence of walkability on sale price of residential properties, through a case study in Rio de Janeiro city. The analyzes were conducted in a hedonic approach, using structural equation models to construct the latent variables of the unobservable characteristics, such as walkability and public safety included in the tasted model. This methodology made it possible to analyze the structure of latent variables, their mutual relationships and their relationship with the real estate values. The results obtained show that square meter price of residential real estate for sale, in the study area, increases as the walkability increases. Public safety, road slope and the presence of beautiful buildings were identified as the key factors to explain walkability and, consequently, to aggregate monetary value for realty.
\end{abstract}

\section{INTRODUÇÃO}

A avaliação econômica de projetos de investimentos urbanos e de transportes inclui a estimativa dos seus benefícios futuros. Esses benefícios podem estar relacionados com o desenvolvimento econômico de uma determinada região, a melhoria da qualidade de vida da população local, a redução dos tempos de viagem dos usuários, a redução de acidentes de trânsito, mitigação de congestionamentos, minimização da poluição gerada entre outros. A quantificação destes benefícios, assim como dos custos associados à implantação dos projetos, é fundamental para uma adequada estimação do retorno desses investimentos. Entretanto, os valores monetários de alguns dos benefícios citados não são facilmente estimados, pois não existe um mer- 
cado formal de compra e venda de poluição ou de qualidade de vida, por exemplo. Existe, entretanto, uma oferta e demanda por esses elementos e, através dessa necessidade implícita, é possível inferir seus respectivos preços de equilíbrio (Hermann e Haddad, 2005). Da mesma forma, não existe um mercado formal para a caminhabilidade. No entanto, projetos urbanos e de transportes que possuam ações que visem melhorar a caminhabilidade de uma região podem culminar em retorno econômico para o setor público e privado.

Por caminhabilidade entende-se a tradução numérica de quão amigável é uma região para atender a demandas diárias de deslocamento, como ir ao trabalho, a escola, lazer entre outros, através de viagens a pé (Gilderbloom et al., 2015; Rauterkus e Miller, 2011; Pivo e Fisher, 2011; Chatman, 2009). 0 conceito pode ser explicado por cinco dimensões utilizadas para descrever elementos físicos, como calçadas e travessias, e elementos percebidos, como segurança pública, conforto e aprazibilidade. São elas: densidade residencial ou populacional, diversidade de uso de solo, desenho urbano, acesso ao destino e disponibilidade de sistemas de transporte público (Ewing e Cervero, 2010a). Formas de atribuir valor a esses elementos individualmente podem identificar o valor do conceito global e assim torna-se possível identificar o impacto econômico dos investimentos em caminhabilidade.

Uma forma de identificar a valoração dos conjuntos de elementos que agregados explicam caminhabilidade é através da avaliação do seu impacto no preço de imóveis residenciais. A comprovação da valorização do imóvel em bairros caminháveis pode guiar investimentos ou planos de ação voltados à construção de um ambiente que estimule as viagens a pé (Bliesner et al., 2010; Cortright, 2009; Gilderbloom et al., 2015; Greene, 2009; Kim, 2015; Pivo e Fisher, 2011; Rauterkus e Miller, 2011; Washington, 2013). Esse enfoque justifica-se, visto que, no mercado imobiliário, a precificação do produto ocorre de forma hedônica, ou seja, não se refere somente à valoração das suas características físicas, mas, à valoração da utilidade percebida pela cesta de atributos que o comprador adquire com a aquisição do bem (Hermann e Haddad, 2005). Ainda, acessibilidade e características do entorno são alguns dos elementos identificados como importantes no processo de escolha da residência (Pivo e Fisher, 2011; Rauterkus e Miller, 2011), chegando, em alguns casos, a ser mais significativo que o próprio preço (Belden Russonello \& Stewart LLC, 2011).

Por fim, os trabalhos identificados que buscam valorar caminhabilidade através do mercado imobiliário, foram desenvolvidos em cidades europeias e americanas que possuem um ambiente urbano construído diferente do encontrado na América Latina. Sendo assim, é importante realizar pesquisas regionalizadas para a correta avaliação do impacto e não foram encontrados trabalhos com este enfoque com aplicação nacional. Adicionalmente, os estudos de preços hedônicos encontrados na literatura utilizam tipicamente modelos de regressões, onde os preços de vendas das unidades residenciais são modelados regredindo em função da medição de seus atributos (Fávero, 2003). Porém, a maioria dos atributos significativos na aquisição de um imóvel é de natureza intangível, tornando necessário utilizar ferramentas que construam variáveis não diretamente observadas através de variáveis observáveis. Para isso, neste estudo, utilizamse modelos de equações estruturais.

Nesse sentido reside a contribuição desse trabalho, cujo objetivo é avaliar o impacto da caminhabilidade no preço dos imóveis residenciais. Por consequência, avaliam-se as variáveis observáveis necessárias para construção do construto da caminhabilidade, através um estudo de caso de uma região da zona norte da cidade do Rio de Janeiro. Ressalta-se que os resultados desta análise podem auxiliar o governo na avaliação de projetos urbanísticos e de transportes. 
Quantificar os benefícios econômicos da caminhabilidade pode influenciar a elaboração de políticas públicas que priorizem a alocação de recursos em infraestruturas que facilitem a circulação de pedestres, justificadas e estimuladas por sua rentabilidade.

\section{CAMINHABILIDADE E O MERCADO IMOBILIÁRIO}

Um bairro caminhável é seguro, bem servido de comércio e serviços e repleto de qualidades que torna agradável a experiência de caminhar (Talen e Koschinsky, 2013). Esses locais são normalmente caracterizados pela alta concentração de lojas de varejo para consumo geral e pessoal e possuem uma série de elementos físicos e percebidos que estimulam os deslocamentos a pé Os investimentos em área que estimulam a realização de viagens por caminhada podem gerar benefícios para o ambiente (pela redução de emissões através do decréscimo de viagens motorizadas), para as pessoas (pelo aumento do nível de atividade física e consequente melhoria na saúde) e para o desenvolvimento econômico que. entre outros fatores, pode ser representado pela valorização imobiliária de comunidades caminháveis (Pérez et al., 2017). A melhoria na caminhabilidade da região é, portanto, um importante recurso econômico e social das comunidades (Gilderbloom et al., 2015; Koschinsky e Talen, 2015; Pivo e Fisher, 2011).

Ewing e Cervero (2010b) propõem que o ambiente construído pode influencia a caminhabilidade através de cinco dimensões conhecidas como 5D’s. Alguns pesquisadores já buscaram identificar a contribuição e cada uma dessas dimensões na valorização de imóveis comercializados. São elas: densidade (W. Li et al., 2014), diversidade (Shen e Karimi, 2017; Song e Knaap, 2004), desenho urbano, acessibilidade ao destino e distância ao transporte público (Cervero e Kang, 2011; Dziauddin et al., 2013; Munoz-Raskin, 2010; Rodriguez e Mojica, 2009). Alguns estudos ainda incluem a administração da demanda por viagem como uma sexta dimensão. Porém, em países em desenvolvimento, a segurança pública surge como um importante fator para estímulo à caminhada (Larrañaga e Strambi, 2014). Jane Jacobs, no seu livro "Morte e vida em grandes cidades" (Jacobs, 2011), originalmente publicado em 1961, já alertava sobre a relação da caminhabilidade com a segurança pública. Jacobs dizia que uso misto do solo e melhorias nas condições das calçadas ajuda a trazer as pessoas para usar a cidade, fora de suas casas. A vivacidade urbana, segundo Jacobs, ajuda a criar o conceito de ter "olhos nas ruas", onde os residentes e transeuntes podem assistir e denunciar atividades criminosas ao longo de todas as horas do dia. A recíproca também é verdadeira. Há indícios de que altas taxas de criminalidade podem inibir atividades físicas para transporte ou lazer (Foster et al., 2014; Lorenc et al., 2014). Explicar a relação entre segurança pública e caminhabilidade é importante para compreender que o incentivo à caminhabilidade não necessariamente precisa estar baseado em medidas físicas, como melhoria das condições das calçadas ou implantação de moderadores de tráfego. Melhorias na condição de segurança podem também incentivar as viagens a pé e as soluções urbanísticas e de mobilidade devem sempre considerar os impactos que sua implementação terá na segurança pública (McDonald, 2008).

Os benefícios do aumento da caminhabilidade vão além de melhoria na condição de saúde e segurança resultando, também, em retornos econômicos que podem estar atrelados ao aumento das vendas, crescimento do número de empregos, redução dos custos de transportes entre outros (Bliesner et al., 2010; Kornas et al., 2017; Litman, 2003, 2010). Uma forma de avaliar esses benefícios é identificando quanto de valorização no preço de venda do imóvel pode ser atribuída às características do ambiente construído favoráveis a caminhabilidade da região. Essa afirmação baseia-se no fato de os consumidores buscarem por imóveis em locais com alta 
acessibilidade (Rahadi et al., 2013; Rauterkus e Miller, 2011), com uso do solo misto (Bitter et al., 2017; Bliesner et al., 2010), boa condição das calçadas (Belden Russonello \& Stewart LLC, 2011) e seguros (Montenegro et al., 2013), valorizando esses atributos na hora da decisão pela compra. Esses resultados demonstram a importância de atributos vinculados à localização na decisão de compra por determinado imóvel e podem representar um desejo indireto por segurança pública e pela possibilidade de deslocar-se a pé. A localização pode ser mais importante que o preço (Salleh et al., 2015) e que o tamanho do imóvel (Belden Russonello \& Stewart LLC, 2011). Estar a 30 minutos do trabalho e ter calçadas e locais para caminhar estão entre as três mais importantes características na escolha da comunidade para residir (Belden Russonello \& Stewart LLC, 2011). Acessibilidade, proximidade de pontos de interesse e topografia favorável, características de regiões com alta caminhabilidade, provocam aumento na demanda e, consequentemente, no valor dos imóveis (Rauterkus e Miller, 2011).

A localização faz parte da cesta de atributos que o comprador adquire juntamente com as chaves de imóveis. No caso imobiliário, esses atributos referem-se às propriedades do imóvel e às amenidades urbanas no contexto local ao qual ele está inserido (H. Li et al., 2016). A satisfação do consumidor com as amenidades urbanas depende se sua percepção sobre o ambiente é positiva ou negativa quanto à localização, condição de trânsito e transporte, poluição, oferta de entretenimento, segurança, entre outros (Hermann e Haddad, 2005).

Conforme a teoria dos preços hedônicos, cada atributo é responsável por adicionar um valor ao preço total do conjunto e esse conjunto pode ser composto de características intrínsecas e extrínsecas que podem ser observadas diretamente e indiretamente (Fávero, 2003). Esses atributos são decisivos no processo de escolha do item que será adquirido e na concretização do negócio, sendo as características do bairro um atributo chave para os compradores. A metodologia busca identificar a máxima utilidade de cada atributo para estimar seu valor em um bem multi-atributos (Rosen, 1974).

Em geral, os modelos baseados na teoria hedônica visam separar os atributos de um bem uns dos outros para efeitos de estimativa de preços implícitos (Shyr et al., 2013). Ao aplica-los, sempre se deve indagar se todos os atributos necessários estão inclusos e se são conhecidas suas propriedades e magnitudes (Hermann e Haddad, 2005). Tipicamente, são utilizadas análises de regressões, onde os preços de vendas das unidades residenciais são regredidos em função da medição de seus atributos (Fávero, 2003). Porém, a natureza intangível da maioria dos atributos exige a utilização de técnicas que lidem com essa particularidade, como os modelos de equações estruturais (Hair et al., 2009).

A maioria dos estudos existentes que já estudaram os efeitos da caminhabilidade no preço de venda dos imóveis, analisam a o efeito através do indicador de caminhabilidade WalkScore®, concebido para atender uma demanda do mercado imobiliário americano (Bliesner et al., 2010; Cortright, 2009; Pivo e Fisher, 2011; Washington, 2013). Ele é calculado com base na distância do imóvel a 13 diferentes categorias de pontos de interesses, como escolas, parques, lojas, entre outros (Carr et al., 2010; Duncan et al., 2011). Ainda, há estudos que consideraram a criação de indicadores próprios de caminhabilidade (Leinberger e Alfonzo, 2007; W. Li et al., 2014; Rauterkus e Miller, 2011) ou pela avaliação do chamado 'Novo Urbanismo', que também traz conceitos de um espaço urbano que favorece a caminhabilidade.

Em todos os estudos citados encontrou-se uma relação positiva entre o indicador e o preço de venda de imóveis, sejam eles residenciais ou comerciais. Os resultados dessas pesquisas sugerem que a caminhabilidade adiciona valor financeiro aos imóveis, o que pode tornar atrativo 
a investidores e tomadores de decisão o desenvolvimento de bairros caminháveis ou a requalificação do ambiente já construído (Rauterkus e Miller, 2011). Esse aumento no valor do imóvel, no entanto, pode contribuir para o aumento da desigualdade social, levando a população mais pobre e com menos condições financeiras a morar em locais menos caminháveis (Mooya, 2011; Riggs e Riggs, 2016; Washington, 2013). Essa tendência de valorização identificada em outros locais deve se refletir no contexto brasileiro. Entretanto, não existe uma métrica clara da caminhabilidade para a realidade nacional que norteie essas políticas e investimentos, e facilitem sua replicabilidade.

\section{DADOS UTILIZADOS}

Para realização do estudo foram combinados dados de uma pesquisa de entrevistas domiciliares realizadas sobre nível de atividade física, conduzida e disponibilizada aos autores pelo WRI Brasil Cidades Sustentáveis; e informações de preços de imóveis à venda na região, obtidas através do mapa de preços do site do ZAP Imóveis. Ainda, foram coletadas e informações socioeconômicas das zonas censitárias na área de estudo apresentada na Figura 1.

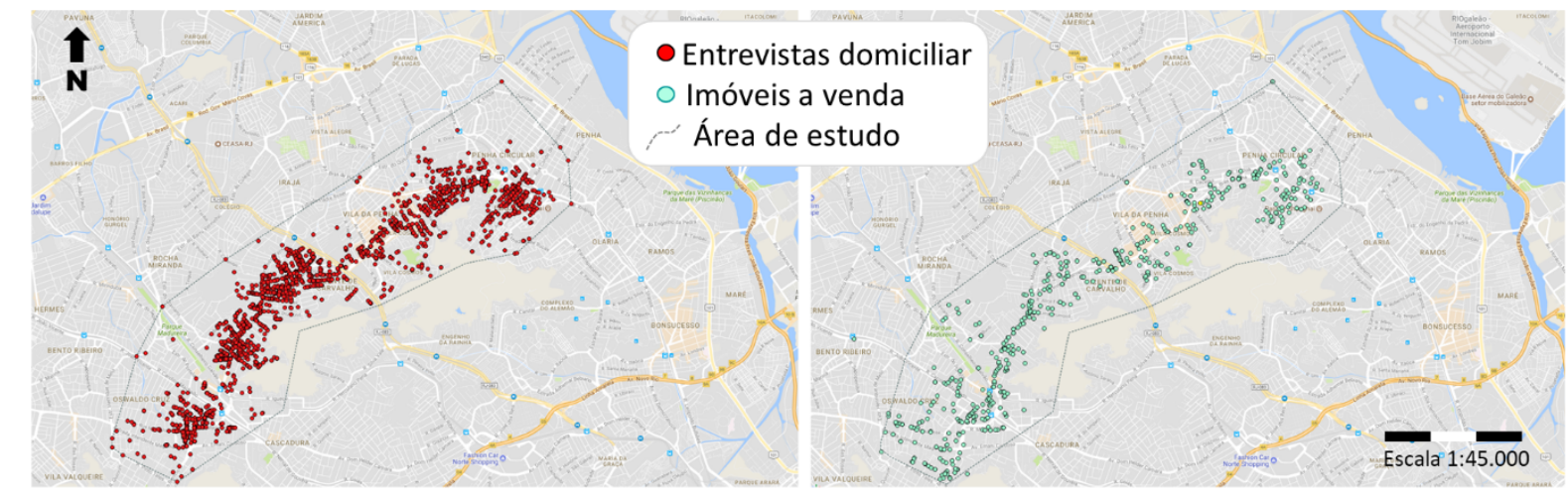

Figura 1: Mapa da área de investigação

O banco de dados proveniente da pesquisa domiciliar disponibilizada é constituído por entrevistas pessoais, individuais e domiciliares em uma amostra de 3000 entrevistados, sendo que 2044 entrevistas foram consideradas completas e válidas para as análises. Foram entrevistados homens ou mulheres, de 18 a 70 anos, alfabetizados, sem limitações cognitivas e sem mobilidade limitada, residentes na área de estudo por tempo mínimo de um ano. As respostas se referiam às percepções dos usuários sobre os itens perguntados e estavam divididas em seis grandes grupos, sendo eles: (i) dados do entrevistado, (ii) meios de transportes, (iii) atividade física de deslocamento, (iv) atividade física no lazer, (v) percepções do ambiente do bairro e (iv) qualidade de vida. As entrevistas foram utilizadas como unidade base de análise, sendo as demais fontes de dados relacionadas aos entrevistados.

Já os dados relativos aos valores dos imóveis na região de estudo foram obtidos através do Mapa de Preços divulgado no site do ZAP Imóveis. Os preços divulgados no site são baseados na série histórica de preços dos imóveis anunciados por imobiliárias ou pessoas físicas no site do ZAP Imóveis, corrigidos pelo índice FipeZap (FIPE, 2011). É premissa deste estudo que apesar dos valores utilizados terem sido corrigidos pelo índice e não representarem efetivamente o preço da transação, eles são uma boa proxy dos valores de venda praticados na região de estudo. Ao total, foram obtidos 572 registros. Como forma de uniformizar a unidade de análise, forma 
utilizados os valores médios dos preços para cada zona censitária dentro da área de estudo. 0 preço médio por zona foi relacionado com cada entrevista individualmente.

\section{MÉTODO}

Os dados provenientes da pesquisa domiciliar foram utilizados para construir as variáveis latentes Caminhabilidade e Seg_Pública. Para testar a teoria de formação dos conceitos e avaliar sua relação com o preço, foram utilizados modelos de equações estruturais (SEM). Os modelos SEM são compostos de um modelo de mensuração e um modelo estrutural. O objetivo do modelo de mensuração é atestar a validade das relações entre as variáveis não observadas (latentes) com os indicadores. Os indicadores geralmente são respostas às perguntas da pesquisa, representando a ligação entre os escores de um instrumento de medida e o construto teórico em estudo o qual os indicadores foram designados a medir. 0 modelo estrutural é o resultado da conversão do modelo de mensuração proposto em um sistema de equações que representam a relação desenhada no modelo de mensuração. 0 modelo estrutural é validado a partir dos coeficientes das equações obtidos pelo diagrama de caminhos.

A equação estrutural (1) e a equação de mensuração (2) no modelo de variável latente podem ser expressas como (Hair et al., 2009):

$$
\begin{aligned}
& \eta=\Gamma X+\zeta \\
& y=\Lambda \eta+\varepsilon
\end{aligned}
$$

onde $\boldsymbol{\eta}$ é um vetor $(M \times 1)$ de variáveis latentes e $\mathbf{X}$ é um vetor $(K \times 1)$ de valores observados para as variáveis exógenas, que constituem as múltiplas causas observáveis. A matriz $\Gamma(\mathrm{M} \times \mathrm{K})$ contém coeficientes de regressão desconhecidos. Na Equação 2, y é um vetor $(\mathrm{P} \times 1)$ de indicadores observáveis de $\boldsymbol{\eta}$ e $\boldsymbol{\Lambda}$ é uma matriz $(\mathrm{P} \times \mathrm{M})$ de cargas fatoriais. Tanto as perturbações estruturais $\zeta(\mathrm{M} \times 1)$ como os erros de medição $\varepsilon(\mathrm{P} \times 1)$ são normalmente distribuídos, mutuamente independentes e admite-se em todas as variáveis um valor esperado zero. Os SEM buscam validar a relação entre construtos de uma forma causal, e não simplesmente correlacional.

A construção do modelo iniciou com a definição dos construtos individuais (variáveis latentes) que expressam a teoria que se deseja confirmar. Na segunda etapa, ocorreu o desenvolvimento do modelo de mensuração. Nessa etapa foi realizada a representação gráfica do modelo (diagrama de caminhos), definindo-se cargas fatoriais fixas das variáveis e seus erros de estimação. Devido ao construto ser uma variável não observada, ele não possui uma dimensão associada e torna-se necessário fornecer uma escala para o cálculo dos coeficientes (Larrañaga, 2005). Fixa-se, portanto, uma das cargas fatoriais com valor igual a 1.

A representação gráfica determina as características do modelo, onde as variáveis observáveis são representadas em retângulos e as não observáveis em elipses; e as relações causais com setas. Setas em uma direção representam as relações causa e efeito e em duas direções indicam covariância ou correlação (Hox e Bechger, 1998). Essa relação de causalidade pode ser determinada de forma reflexiva ou formativa. Modelos reflexivos representam teorias em que a variável latente exerce influência sobre as variáveis observadas. As variáveis precisam ser altamente correlacionadas visto que todas são influenciadas por um fator comum. 0 erro de mensuração está associado às variáveis observadas, representando as dimensões nas quais a variável latente não consegue explicá-las. Nos modelos formativos, os indicadores são a causa da variável latente. As variáveis não precisam ser necessariamente correlacionadas, mas todas as variáveis importantes para descrever a variável latente devem estar inclusas no modelo. 0 erro de mensuração, nesse caso, deve estar vinculado à variável latente, explicando a imprecisão das 
variáveis de formar o construto (Hair et al., 2009). O produto da análise é uma série de equações representativas das relações desenhadas entre variáveis observadas e latentes e entre duas variáveis latentes e seus coeficientes

Construído o modelo, testa-se a teoria. Para modelos SEM, verifica-se as razões críticas (CR) de cada relação entre variável dependente e causa, e os índices GFI (Goodness-of-Fit Index) e RMSEA (Root Mean Square Error of Aproximation) para o ajuste do modelo como um todo. A razão crítica CR é obtida pela estimativa do parâmetro dividida pelo seu erro padrão (Larrañaga, 2005). Assume-se que a estimativa será normalmente distribuída, e, portanto, valores de CR acima de 1.96 são considerados significativos a um nível de confiabilidade de $5 \%$ (Schumacker e Lomax, 2010). 0 parâmetro GFI, referente ao ajuste do modelo total, avalia se a matriz estimada reproduz a quantidade de variância e covariância da matriz observada (Larrañaga, 2005) sendo valores próximos de 1 os melhores ajustes (Hair et al., 2009). Complementarmente, foi avaliado o valor do parâmetro RMSEA que busca explicar quão bem o modelo se ajusta a uma população e não somente a uma amostra. Valores abaixo de 0.10 são considerados aceitáveis (Hair et al., 2009).

\section{DESENVOLVIMENTO E ANÁLISE DOS MODELOS}

A variável latente Segurança_pública agregou todas as variáveis que avaliavam as percepções de segurança na pesquisa domiciliar. Para a variável que foi denominada de Caminhabilidade, as variáveis agregadas foram escolhidas com base nas cinco dimensões do ambiente construído que representam caminhabilidade (5D’s) propostas por Ewing e Cervero (2010). A Tabela 1 apresenta as variáveis e dimensões consideradas.

Tabela 1: Variáveis relativas às cinco dimensões da caminhabilidade utilizadas

\begin{tabular}{|c|c|c|}
\hline Dimensão & Variável Observada & Afirmativas do Questionário \\
\hline Densidade & Muitas_residencias & $\begin{array}{l}\text { Sua residência está localizada em um bairro com muitas } \\
\text { outras residências. }\end{array}$ \\
\hline \multirow[t]{2}{*}{$\begin{array}{l}\text { Diversidade de uso do solo } \\
\text { e acesso ao destino }\end{array}$} & Muitos_comercios & $\begin{array}{l}\text { Sua residência está localizada em um bairro com muitos } \\
\text { prédios comerciais, indústrias e escritórios. }\end{array}$ \\
\hline & Construções bonitas & Existem muitas construções/casas bonitas no seu bairro. \\
\hline \multirow[t]{3}{*}{ Desenho urbano } & Caminhos_alternativos & $\begin{array}{l}\text { Existem caminhos alternativos que você possa usar para ir de } \\
\text { um lugar para outro no seu bairro. }\end{array}$ \\
\hline & Declividade & $\begin{array}{l}\text { As ruas do seu bairro têm subidas e descidas que dificultam } \\
\text { caminhar ou andar de bicicleta }\end{array}$ \\
\hline & Travessia_pedestres & $\begin{array}{l}\text { Existem faixas, sinais ou passarelas que facilitam a travessia } \\
\text { das ruas movimentadas do seu bairro. }\end{array}$ \\
\hline $\begin{array}{l}\text { Disponibilidade do } \\
\text { Transporte Público }\end{array}$ & Satisf_acesso_transp_público & $\begin{array}{l}\text { Você está satisfeito com o acesso ao transporte público no } \\
\text { seu bairro? }\end{array}$ \\
\hline
\end{tabular}

Os primeiros modelos testados apresentaram coeficientes negativos para as varáveis Muitos_comerc e Caminhos_alterantivos, indicando que estes fatores piorariam a condição de caminhabilidade da região. A principal justificativa para o sinal contrário ao esperado da variável Muitos_comerc refere-se à própria questão de pesquisa. Os entrevistados deveriam responder se concordavam ou não com a afirmação "Sua residência está localizada em um bairro com muitos prédios comerciais, indústrias e escritórios". A sentença inclusa no questionário não prioriza a avaliação da quantidade de comércios existentes. Os entrevistados 
deveriam responder sobre sua concordância em relação a estabelecimentos bastante distintos, tanto em relação ao tipo de uso, quanto às suas relações com a caminhabilidade do bairro. Bairros com muitos prédios comerciais e de escritórios são mais favoráveis à realização de viagens a pé do que bairros essencialmente industriais. As respostas do questionário, sendo assim, não permitiram identificar com clareza a influência dos comércios na caminhabilidade dos bairros. Ressalta-se que a pesquisa foi cedida aos autores quando já realizada, não sendo possível a estes alterar a questão para a obtenção de melhores resultados.

Quanto a variável Caminhos_alterantivos, entende-se que apesar de, na média, os entrevistados concordarem com a afirmativa de existir diferentes opções de rota para acesso ao destino no bairro, a configuração viária da região não possibilita diferentes conexões. Não existe um padrão claro da malha e a topografia impossibilitaria pequenos desvios sem grandes dispêndios de energia. Sendo assim, apesar de ser possível realizar desvios de rota, esses desvios aumentariam as distâncias caminhadas e elas seriam realizadas com mais sacrifício, piorando, dessa forma, a condição de caminhabilidade. Entende-se que as duas variáveis discutidas (Muitos_comerc e Caminhos_alterantivos) são importantes para representar as cinco dimensões do ambiente construído que influencia a caminhabilidade, todavia, não se deseja que um erro de medição ou de especificação perturbe os resultados gerais do modelo (Ortúzar e Willumsen, 2011).

\subsection{Construção do modelo SEM}

A Figura 2 apresenta o modelo final construído. As representações gráficas através do diagrama de caminhos, assim como a estimação do modelo, foram realizadas no software IBP SPSS Amos 24. A variável Caminhabilidade foi construída de forma formativa, pois os autores entendem que as variáveis que representam as dimensões da caminhabilidade é que formam o conceito. No caso do construto que indica a segurança pública entende-se que as percepções de segurança de dia, à noite, no embarque e quanto ao número de crimes são um reflexo da segurança pública como um todo e, portanto, a variável latente Seg_pública, deve ser modelada de forma reflexiva. Com a inserção da variável observada e identificando a relação causal entre as variáveis latentes, característica da modelagem de equações estruturais, são eliminados os problemas de identificação que a configuração poderia ocasionar.

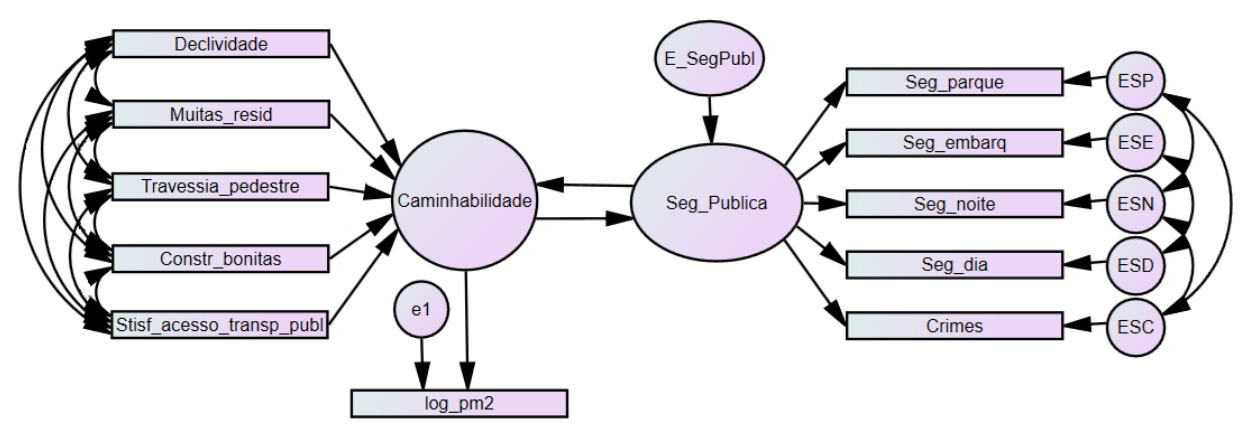

Figura 2: Representação gráfica do modelo de equações estruturais testado

Neste trabalho, a variável dependente foi considerada na forma logarítmica por resultar em um melhor ajuste no modelo final. Conforme hipóteses de pesquisa a serem testadas, foi determinada uma relação direta da Caminhabilidade no log_preço_m2. A relação entre Caminhabilidade e Seg_pública foi analisada como uma relação biunívoca, visto que a caminhabilidade pode 
afetar a segurança pública, pois proporciona vivacidade e aprazibilidade às ruas do bairro, e a segurança pública também pode afetar a caminhabilidade, visto que a sensação de segurança pode incentivar os deslocamentos a pé.

\subsection{Análises estatísticas e resultados do modelo SEM}

Como resultado, o modelo gera o conjunto de equações estruturais descritos entre as equações (3) a (10):

$$
\begin{aligned}
& \text { Caminhabilidade }=0.217 \text { Muitas_resid }+0.344 \text { Constr_bonitas }+ \\
& 0.169 \text { Travessia _ pedestre +0.118Satisf_acesso_transp_publ - } \\
& 0.365 \text { Declividade }+0.390 \text { Seg_Publica }+ \text { ESeg_publ } \\
& \text { Crimes }=-0.449 S_{\text {Seg }} \text { publica }+ \text { ESC } \\
& \text { Seg_dia }=0.771 S e g_{\text {_ }} \text { publica }+E S D \\
& \text { Seg_noite }=0.441 S e g_{\text {_ }} \text { publica }+E S N \\
& \text { Seg_embarq }=0.866 \text { Seg _ publica }+E S E \\
& \text { Seg _ parque }=0.620 S_{\text {Seg }} \text { publica }+E S P \\
& \text { Seg_Pública }=0.512 \text { Caminhabilidade } \\
& \log _{-} \text {pm } 2=0.156 \text { Caminhabilidade }+e
\end{aligned}
$$

\begin{tabular}{|c|c|c|c|c|c|c|c|}
\hline Variável Observada & $\begin{array}{l}\text { Relação } \\
\text { Causal }\end{array}$ & Variável Latente & Pesos & $\begin{array}{l}\text { Pesos } \\
\text { Estandard. }\end{array}$ & E.P & C.R. & $\mathbf{P}$ \\
\hline Muitas_resid & $\rightarrow$ & Caminhabilidade & 0.004 & 0.217 & 0.001 & 3.485 & $* * *$ \\
\hline Constr_bonitas & $\rightarrow$ & Caminhabilidade & 0.006 & 0.344 & 0.001 & 4.213 & $* * *$ \\
\hline Travessia_pedestre & $\rightarrow$ & Caminhabilidade & 0.003 & 0.169 & 0.001 & 3.500 & $* * *$ \\
\hline Satisf_acesso_transp_publ & $\rightarrow$ & Caminhabilidade & 0.003 & 0.118 & 0.001 & 3.027 & 0.002 \\
\hline Declividade & $\rightarrow$ & Caminhabilidade & -0.006 & -0.365 & 0.001 & -4.387 & $* * *$ \\
\hline Crimes & $\leftarrow$ & Seg_Publica & -0.449 & -0.305 & 0.034 & -13.35 & $* * *$ \\
\hline Seg_dia & $\leftarrow$ & Seg_Publica & 1.315 & 0.771 & 0.083 & 15.895 & $* * *$ \\
\hline Seg_noite & $\leftarrow$ & Seg_Publica & 0.658 & 0.441 & 0.033 & 19.805 & $* * *$ \\
\hline Seg_embarq & $\leftarrow$ & Seg_Publica & 1.984 & 0.866 & 0.083 & 16.637 & $* * *$ \\
\hline Seg_parque & $\leftarrow$ & Seg_Publica & 1 & 0.620 & - & - & - \\
\hline log_pm2 & $\leftarrow$ & Caminhabilidade & 1 & 0.156 & - & - & - \\
\hline Variável latente (efeito) & $\begin{array}{l}\text { Relação } \\
\text { causal }\end{array}$ & $\begin{array}{l}\text { Variável latente } \\
\text { (causa) }\end{array}$ & Pesos & $\begin{array}{l}\text { Pesos } \\
\text { Estandard. }\end{array}$ & E.P & C.R. & $P$ \\
\hline Seg_Publica & $\leftarrow$ & Caminhabilidade & 16.024 & 0.512 & 2.635 & 60.81 & $* * *$ \\
\hline Caminhabilidade & $\leftarrow$ & Seg_Publica & 0.012 & 0.390 & 0.005 & 2,307 & 0.021 \\
\hline \multicolumn{3}{|c|}{ Número de parâmetrôs estimado } & \multicolumn{4}{|c|}{37} & \\
\hline \multicolumn{3}{|c|}{ Graus de liberdade } & \multicolumn{4}{|c|}{29} & \\
\hline \multicolumn{3}{|c|}{ GFI } & \multicolumn{4}{|c|}{0.965} & \\
\hline \multicolumn{3}{|c|}{ RMSEA } & \multicolumn{3}{|c|}{0.049} & & \\
\hline
\end{tabular}

0 modelo é significativo estatisticamente, como é possível observar na Tabela 2 e, portanto, valida a teoria proposta com sinais dos coeficientes de cada indicador condizentes ao previsto. O mesmo ocorre com as variáveis latentes Seg_publica e Caminhabilidade, que apresentaram uma forte relação positiva nas duas direções comprovando a teoria de que a segurança pública possui uma relação causal mútua com a caminhabilidade. Esse resultado está de acordo com o encontrado em outros estudos referenciados neste trabalho (Gilderbloom et al., 2015; W. Li et al., 2014; Pivo e Fisher, 2011).

Tabela 2: Resultados da estimação do modelo de equações estruturais ajustado 
Analisando as relações de cada variável latente com as variáveis observáveis, nota-se que variáveis observadas que sofrem maior influência da Seg_Publica são as variáveis que indicam a percepção de segurança ao embarcar e desembarcar do transporte público (0.866) e a que representa a segurança em caminhar ou andar de bicicleta de dia no bairro (0.771). Esse resultado pode ser explicado pelo perfil de deslocamento dos entrevistados, onde $68 \%$ relatam realizar algum deslocamento a pé nas suas viagens diárias e 57\% utilizam transporte público, sendo esses usuários mais sensíveis à segurança no transporte.

Para formação da variável latente Caminhabilidade, as variáveis mais influentes foram a variável latente Seg_pública (0.39), seguido pelas variáveis observadas Declividade (-0.365) e Constr_bonitas (0.344). Ressalta-se, com esse resultado, que a condição de segurança é um importante fator na escolha do modo de transporte e está de acordo com outros estudos sobre o tema realizados no Brasil (Larrañaga, Cybis, Arellana, et al., 2015) . Ainda, segundo a The Social Progress Imperative (2016), o Brasil é o 123ํㅜㄹ país no ranking global de segurança pessoal, sendo um dos piores colocados da América do Sul. Esperava-se, portanto, que esta variável apresentasse importância significativa na construção do construto latente e resultado positivo na formação da Caminhabilidade.

A influência da caminhabilidade no preço por $\mathrm{m}^{2}$ dos imóveis apresentou coeficiente positivo, confirmando a hipótese prévia da influência da caminhabilidade na valoração dos imóveis. 0 valor do coeficiente da regressão parametrizado é apresentado na Tabela 2, com valor igual a 0.156. A relação positiva indica que quanto mais caminhável é a região, mais valorizados são os imóveis residenciais disponíveis para venda. Esse resultado é condizente com os resultados encontrados por diversos autores que já estudaram essa relação (Bartholomew e Ewing, 2011; Cortright, 2009; Gilderbloom et al., 2015; Kim, 2015; Leinberger e Alfonzo, 2007; Pivo e Fisher, 2011; Rauterkus e Miller, 2011; Washington, 2013).

\subsection{Efeito marginal de alternativas de melhoria da caminhabilidade}

Para medir o impacto da caminhabilidade no preço dos imóveis, foram calculados os efeitos marginais das variáveis formativas no preço por $\mathrm{m}^{2}$ dos imóveis residenciais. Os efeitos foram calculados alterando o valor de cada variável no valor correspondente a um incremento em uma unidade na sua escala de mensuração. As variáveis utilizadas são variáveis ordinais, com quatro categorias, variando da concordância total a discordância total. Assim, um incremento em uma categoria representa um incremento de $25 \%$ na variável. Os resultados apresentados na Tabela 3 , representam o impacto no preço por $\mathrm{m}^{2}$ no aumento de $25 \%$ no valor da variável medida. A equação 11 apresenta a formulação para o cálculo do efeito marginal. A exponencial é necessária devido à variável dependente ter sido utilizada na forma logarítmica.

$$
\frac{P_{0}}{P_{i}}=e^{0.25^{*} \lambda \text { var iavel* } \lambda \text { camin habilidade }}
$$

Tabela 3: Efeito marginal do preço por $\mathrm{m}^{2}$

\begin{tabular}{llll}
\hline Varíaveis & $\mathbf{1}$ incremento (25\%) & $\mathbf{2}$ incrementos (50\%) & 3 incrementos (75\%) \\
\hline Seg_Publica & $1.53 \%$ & $3.09 \%$ & $4.67 \%$ \\
Declividade & $1.48 \%$ & $2.99 \%$ & $4.51 \%$ \\
Constr_bonitas & $1.40 \%$ & $2.82 \%$ & $4.26 \%$ \\
Muitas_resid & $0.89 \%$ & $1.79 \%$ & $2.70 \%$ \\
Travessia_pedestre & $0.68 \%$ & $1.37 \%$ & $2.06 \%$ \\
Satisf_acesso_transp_publ & $0.48 \%$ & $0.96 \%$ & $1.44 \%$ \\
\hline
\end{tabular}


Os efeitos calculados para segurança pública são referentes à percepção geral da condição de segurança pública no bairro. Ações que façam as pessoas sentirem-se mais seguras como maior presença de patrulhamento, melhoria na iluminação pública e o próprio aumento da caminhabilidade, podem resultar em uma valorização dos imóveis disponíveis para a venda. 0 aumento no número de residências pode ser incentivado com revisões nos planos diretores e políticas de zoneamento, através da alteração dos índices construtivos e criação de políticas de habitação social. A existência de travessia para pedestres também é dependente de investimentos públicos. Além de simples, é uma medida barata para impactar positivamente a caminhabilidade. Por fim, o acesso ao transporte público também pode ser melhorado com o aumento do número de travessias, melhoria nas condições dos passeios, rampas de acessibilidade, aumento no número de paradas, na disponibilidade de sistemas ofertados entre outras soluções. É mais custoso para o poder público propor melhorias que incrementem a percepção do usuário para as variáveis Declividade e Constr_bonitas. A declividade é função da topografia da região e do perfil longitudinal da via, sendo difícil a alteração do seu valor. A aparência das construções pode ser melhorada através de incentivos públicos, mas é atribuição do dono do imóvel particular melhorar o aspecto estético da construção e investir na sua boa aparência e beleza.

Verificou-se também o impacto da variável latente Caminhabilidade no preço. A avaliação do impacto diretamente na variável auxilia no estudo do efeito combinado dos incrementos das variáveis formativas. Os resultados indicam que para obter um percentual de valorização do imóvel superior a 5\%, as melhorias na caminhabilidade da região deveriam ser superiores a $30 \%$. Ao aplicar essa valorização no preço do $\mathrm{m}^{2}$ médio da amostra ( $\mathrm{R} \$ 4.031,08$ ), foi possível comparar monetariamente os ganhos que podem ser obtidos. Para um imóvel de $100 \mathrm{~m}^{2}, 30 \%$ de aumento na caminhabilidade podem resultar em 19 mil reais a mais no preço final do imóvel. Embora, por sua natureza, este incremento percentual nas variáveis latentes seja de difícil mensuração, o valor marginal das variáveis deste estudo proporciona bons indicativos sobre ações promotoras da valorização urbana. Ou seja, para alcançar 30\% de aumento na caminhabilidade é necessário combinar melhorarias na percepção dos residentes sobre a segurança pública, a declividade do bairro, o acesso ao transporte público, a beleza das construções, o número de residências e o número e qualidade das travessias de pedestres.

\section{CONSIDERAÇÕES FINAIS}

Neste trabalho, melhorar as condições de caminhabilidade significa melhorar a percepção dos usuários quanto ao número de residências, à beleza das construções, ao número de travessias de pedestres, ao acesso ao transporte público, à declividade das vias e à segurança púbica da região. As análises são válidas para uma condição local e influenciadas pelas características do ambiente construído e pelo padrão de transporte da população local. Conforme comentado, algumas percepções são difíceis de alterar pois elas refletem características do ambiente que não são atribuição do poder público ou dos interessados em promover melhorias. É interessante observar, entretanto, que a mudança na caminhabilidade pode surgir de uma combinação de pequenas alterações em cada uma das variáveis observadas.

A segurança pública, o fator mais importante na explicação da caminhabilidade nesse estudo poderia e deveria ser melhorada. Embora os problemas de segurança na cidade do Rio de Janeiro tenham razões socioeconômicas intrínsecas e complexas, as medidas de melhoria urbanísticas e do ambiente construído podem contribuir para aumentar a percepção de segurança. Esse problema foi abordado em outras cidades com medidas como iluminação (Welsh e 
Farrington, 2008) e paisagismo (Donovan e Prestemon, 2012; Kuo e Sullivan, 2001) como parte da prevenção do crime através do desenho urbano (Crime Prevention through Environmental Design - CPTED) (P. Cozens e Love, 2015; P. M. Cozens, 2008; Fennelly e Crowe, 2013).

Ressalta-se que os resultados indicam que as características dos bairros, favoráveis a caminhada, possuem, efetivamente, influência sobre o preço de venda de imóveis residenciais. É possível inferir, por essa constatação, que as razões pelas quais os indivíduos escolhem seu local de moradia não são mais baseadas no uso de transporte individual motorizado. As externalidades do transporte - como congestionamento, tempo de deslocamento, alto custo de combustível guiaram a procura de imóveis em locais que propiciem fácil acesso aos pontos de interesse. Essa constatação ressalta a necessidade de adequação das políticas de desenvolvimento em prol da caminhabilidade, pois investimentos que tornam os bairros menos caminháveis podem estar na contramão da demanda dos consumidores (Washington, 2013). Ainda, é necessário ressaltar que valorização imobiliária pode significar que pessoas com baixo poder aquisitivos sejam afastadas de regiões com alta caminhabilidade Medidas preventivas para evitar que processos de melhoria do ambiente acarretem desagregação social devem ser consideradas em todos os projetos urbanísticos.

Para o mercado imobiliário nacional, apresenta-se a oportunidade de implantar um indicador de caminhabilidade, como já acontece em larga escala nos Estados Unidos com o WalkS-

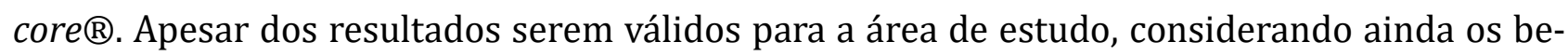
nefícios econômicos descritos, a caminhabilidade deve estar integrada nos planos estratégicos de desenvolvimento das cidades, planejando e projetando para uma forma mais sustentável de uso do ambiente urbano.

\section{AGRADECIMENTOS}

Os autores agradecem ao WRI Brasil Cidades Sustentáveis pela disponibilização da pesquisa domiciliar utilizada e a ANPET pelo prêmio ANPET de Produção Científica 2017.

\section{REFERÊNCIAS}

Bartholomew, K., e Ewing, R. (2011) Hedonic Price Effects of Pedestrian- and Transit-Oriented Development. Journal of Planning Literature, 26(1), 18-34. DOI: 10.1177/0885412210386540

Belden Russonello \& Stewart LLC. (2011) The 2011 Community Preference Survey What Americans Are Looking for When Deciding Where to Live. Opinion Research Strategic Communication. Washington DC.

Bitter, C., e Krause, A. (2017) The influence of urban design packages on home values. International Journal of Housing Markets and Analysis, 10(2), 184-203. doi:10.1108/IJHMA-08-2015-0049

Bliesner, J., Bouton, S., e Schultz, B. (2010) Walkable Neighborhoods: An Economic Development Strategy., 29. Obtido de http://www.aarp.org/content/dam/aarp/livable-communities/act/transportation/Walkable-Neighborhoods-AnEconomic-Development-Strategy-AARP.pdf

Carr, L. J., Dunsiger, S. I., e Marcus, B. H. (2010) Walk Score as a global estimate of neighborhood walkability. American Journal of Preventive Medicine, 39(5), 460-463. DOI:10.1016/j.amepre.2010.07.007

Cervero, R., e Kang, C. D. (2011) Bus rapid transit impacts on land uses and land values in Seoul, Korea. Transport Policy, 18(1), 102-116. DOI:10.1016/j.tranpol.2010.06.005

Chatman, D. G. (2009) Residential choice, the built environment, and nonwork travel: Evidence using new data and methods. Environment and Planning A, 41(5), 1072-1089. doi:10.1068/a4114

Cortright, J. (2009) Walking the Walk: How Walkability Raises Home Values in U.S. Cities. CEOs for Cities, 1-30. Chicago. DOI: 10.1187/cbe.06-05-0163

Cozens, P., e Love, T. (2015) A Review and Current Status of Crime Prevention through Environmental Design (CPTED). Journal of Planning Literature, 30(4), 393-412. DOI:10.1177/0885412215595440

Cozens, P. M. (2008) New Urbanism, Crime and the Suburbs: A Review of the Evidence. Urban Policy and Research, 26(4), 429444. doi:10.1080/08111140802084759

Donovan, G. H., e Prestemon, J. P. (2012) The Effect of Trees on Crime in Portland, Oregon. Environment and Behavior, 44(1), 3-30. DOI:10.1177/0013916510383238 
Duncan, D. T., Aldstadt, J., Whalen, J., Melly, S. J., e Gortmaker, S. L. (2011) Validation of Walk Score?? for estimating neighborhood walkability: An analysis of four US metropolitan areas. International Journal of Environmental Research and Public Health, 8(11), 4160-4179. D0I:10.3390/ijerph8114160

Dziauddin, M. F., Alvanides, S., e Powe, N. (2013) Estimating the effects of light rail transit (LRT) system on the property values in the Klang Valley, Malaysia: A hedonic house price approach. Jurnal Teknologi (Sciences and Engineering), 61(1), 35-47. DOI:10.11113/jt.v61.1620

Ewing, R., e Cervero, R. (2010a) Travel and the Built Environment. Journal of the American Planning Association, 76 no.3(April 2013), 265-294. DOI:10.1080/01944361003766766

Ewing, R., e Cervero, R. (2010b) Travel and the built environment: a synthesis. Transportation Research Record, 1780(Paper No. 01-3515), 87-114. DOI:10.3141/1780-10

Fávero, L. P. L. (2003) Modelos de preços hedônicos aplicados a imóveis residenciais em lançamento no município de São Paulo. Universidade de São Paulo.

Fennelly, L., e Crowe, T. (2013) Crime prevention through environmental design. (Third edit.). Elsevier, Oxford.

FIPE. (2011) Índice FIPEZAP de preços deimóveis anunciados - Notas Metodológicas. São Paulo.

Foster, S., Knuiman, M., Villanueva, K., Wood, L., Christian, H., e Giles-Corti, B. (2014) Does walkable neighbourhood design influence the association between objective crime and walking? The international journal of behavioral nutrition and physical activity, 11(1), 100. DOI:10.1186/s12966-014-0100-5

Gilderbloom, J. I., Riggs, W. W., e Meares, W. L. (2015) Does walkability matter? An examination of walkability's impact on housing values, foreclosures and crime. Cities, 42,13-24. DOI:10.1016/j.cities.2014.08.001

Greene, J. (2009) Sustainability Focused Data Analysis - To what extent do walkability, crime, and neighborhood predict housing prices? Oregon.

Hair, J. F., Black, W. C., Babin, B. J., Anderson, R. E., e Tatham, R. L. (2009) Análise Multivariada de Dados. (6a edição.). Bookman, Porto Alegre.

Hermann, B. M., e Haddad, E. A. (2005) Mercado Imobiliário e Amenidades Urbanas: A View Through the Window. EST. ECON, 36(2), 237-269. DOI: 10.1590/S0101-41612005000200001

Hox, J. J., e Bechger, T. M. (1998) An Introduction to Structural Equation Modeling. Family Science Review, 11, 354-373. DOI:10.1080/10705510903008345

Jacobs, J. (2011) Morte e vida de grandes cidades. (2 ed). WMF Martins Fontes.

Kim, J. (2015) The Impact of New Urbanism on Single Family Housing Values : The Case of Issaquah Highlands. University of Washington.

Kornas, K., Bornbaum, C., Bushey, C., e Rosella, L. (2017) Exploring active transportation investments and associated benefits for municipal budgets: a scoping review. Transport Reviews, 37(4), 465-487. DOI:10.1080/01441647.2016.1252446

Koschinsky, J., e Talen, E. (2015) Affordable housing and walkable neighborhoods: A national urban analysis. Cityscape: A Journal of Policy Development and Research, 17(2), 13-56. DOI: 10.1108/17506200710779521

Kuo, F. E., e Sullivan, W. C. (2001) ENVIRONMENT AND CRIME IN THE INNER CITY Does Vegetation Reduce Crime? Environment and Behavior, 33(3), 343-367. DOI:10.1177/0013916501333002

Larrañaga, A. M. (2005) Relacionamento entre a forma urbana e as viagens a pé. Universidade Federal do Rio Grande do SUl.

Larrañaga, A. M., Cybis, H. B. B., Arellana, J., Rizzi, L. I., e Strambi, O. (2015) Estimando a importância de características do ambiente construído para estimular bairros caminháveis usando. XXIX CONGRESSO NACIONAL DE PESQUISA EM TRANSPORTE DA ANPET (p. 1946-1958). OURO PRETO. DOI:https://doi.org/10.14295/transportes.v24i2.1091

Larrañaga, A. M., Cybis, H. B. B., e Torres, T. B. (2015) Influência da estrutura urbana na decisão de realizar viagens a pé em Porto Alegre. Transportes, 23(4), 89. DOI:10.14295/transportes.v23i4.924

Larrañaga, A. M., e Strambi, O. (2014) Determinação da importância relativa dos atributos do bairro que estimulam as viagens a pé. XXVIII Congresso de Pesquisa e Ensino em Transportes ANPET.

Leinberger, C. B., e Alfonzo, M. (2007) Walk This Way:The Economic Promise of Walkable Places in Metropolitan Washington, D.C. American School \& University, 79(10), 44-47. Obtido de http://search.proquest.com/docview/61934522?accountid=14695

Li, H., Dennis, Y., Yu, Z., e Tian, G. (2016) Amenity , accessibility and housing values in metropolitan USA : A study of Salt Lake County , Utah. JCIT, 59, 113-125. DOI:10.1016/j.cities.2016.07.001

Li, W., Joh, K., Lee, C., Kim, J.-H., Park, H., e Woo, A. (2014) From Car-Dependent Neighborhoods to Walkers' Paradise. Transportation Research Record: Journal of the Transportation Research Board, 2453(October 2015), 162-170. DOI:10.3141/2453-20

Litman, T. (2003) Economic Value of Walkability. Transportation Research Record, 1828(1), Alexander, C., Ishikawa, S., \& Silverstein, M. (19. DOI:10.3141/1828-01

Litman, T. (2010) Quantifying the Benefits of Nonmotorized Transportation For Achieving Mobility Management Objectives by. Transportation Research Record, 134-140.

Lorenc, T., Petticrew, M., Whitehead, M., Neary, D., Clayton, S., Wright, K., Thomson, H., Cummins, S., Sowden, a, e Renton, a. (2014) Crime, Fear of Crime and Mental Health: Synthesis of Theory and Systematic Reviews of Interventions and Qualitative Evidence. Public Health Research, 2(2), Online publication. DOI:10.3310/phr02020 <http://dx.doi.org/10.3310/phr02020>) 
McDonald, N. C. (2008) The effect of objectively measured crime on walking in minority adults. American Journal of Health Promotion, 22(6), 433-436. DOI:10.4278/ajhp.22.6.433

Montenegro, A., Cristina, E., Angelim, D. S., Martins, R., Rosa, A., Duarte, C. L. M., Maneschy, C. E. A., Martins, D., e Haddad, E. (2013) The influence of urban violence and land title irregularity on the market value of properties : A case study in Belém , an Amazon metropolis., 35, 147-155. DOI:10.1016/j.cities.2013.07.006

Mooya, M. M. (2011) Making urban real estate markets work for the poor : Theory, policy and practice. Cities, 28(3), 238-244. DOI:10.1016/j.cities.2010.09.006

Munoz-Raskin, R. (2010) Walking accessibility to bus rapid transit: Does it affect property values? The case of Bogot??, Colombia. Transport Policy, 17(2), 72-84. DOI:10.1016/j.tranpol.2009.11.002

Ortúzar, J. D. D., e Willumsen, L. G. (2011) Modelling Transport. Modelling Transport. DOI:10.1002/9781119993308

Pérez, K., Olabarria, M., Rojas-Rueda, D., Santamariña-Rubio, E., Borrell, C., e Nieuwenhuijsen, M. (2017) The health and economic benefits of active transport policies in Barcelona. Journal of Transport \& Health, 4, 316-324. DOI:10.1016/j.jth.2017.01.001

Pivo, G., e Fisher, J. D. (2011) The Walkability Premium in Commerical Real Estate Investments. Real Estate Economics, 39(2), 185-219. DOI:10.1111/j.1540-6229.2010.00296.x

Rahadi, R. A., Wiryono, S. K., Koesrindartoto, D. P., e Syamwil, I. B. (2013) Attributes Influencing Housing Product Value and Price in Jakarta Metropolitan Region. Procedia - Social and Behavioral Sciences, 101, 368-378. DOI:10.1016/j.sbspro.2013.07.211

Rauterkus, S. Y. R., e Miller, N. G. (2011) Residential Land Values and Walkability. JOSRE, 3, 23-43. DOI: 10.5555/jsre.3.1.033722n763487886

Riggs, W., e Riggs, W. (2016) Inclusively walkable : exploring the equity of walkable housing in the San Francisco Bay Area., 9839(August). DOI:10.1080/13549839.2014.982080

Rodriguez, D. A., e Mojica, C. H. (2009) Capitalization of BRT network expansions effects into prices of non-expansion areas. Transportation Research Part A: Policy and Practice, 43(5), 560-571. DOI:10.1016/j.tra.2009.02.003

Rosen, S. (1974) Hedonic Prices and Implicit Markets: Product Differentiation in Pure Competition. The Journal of Political Economy, 82(1), 34-55. DOI: 10.1086/260169

Salleh, N. A., Zoher, S. A., Mahayuddin, S. A., e Abdul, Y. (2015) Influencing Factors of Property Buyer in Hillside Residential Development. Procedia - Social and Behavioral Sciences, 170, 586-595. D0I:10.1016/j.sbspro.2015.01.060

Schumacker, R. E., e Lomax, R. G. (2010) A beginner's guide to structural equation modeling. DOI:10.1002/9781118133880.hop202023

Shen, Y., e Karimi, K. (2017) The economic value of streets: mix-scale spatio-functional interaction and housing price patterns. Applied Geography, 79(October), 187-202. DOI:10.1016/j.apgeog.2016.12.012

Shyr, O., Andersson, D. E., Wang, J. M., Huang, T. W., e Liu, O. (2013) Where Do Home Buyers Pay Most for Relative Transit Accessibility? Hong Kong, Taipei and Kaohsiung Compared. Urban Studies, 50(12), 2553-2568. DOI:10.1177/0042098012474510

Song, Y., e Knaap, G. J. (2004) Measuring the effects of mixed land uses on housing values. Regional Science and Urban Economics, 34(6), 663-680. DOI:10.1016/j.regsciurbeco.2004.02.003

Talen, E., e Koschinsky, J. (2013) The Walkable Neighborhood: A Literature Review. International Journal of Sustainable Land Use and Urban Planning, 1(1), 42-63. D0I:10.24102/ijslup.v1i1.211

The Social Progress Imperative. (2016) 2016 Social Progress Index.

Washington, E. (2013) Role of Walkability in Driving Home Values. Leadership and Management in Engineering, 13(3), 123130. DOI: 10.1061/(ASCE)LM.1943-5630.0000222

Welsh, B. C., e Farrington, D. P. (2008) Effects of improved street lighting on crime: a systematic review. Campbell Systematic Reviews, (13), 59. DOI:10.4073/csr.2008.13 